\title{
Correlation between thymidylate synthase and dihydropyrimidine dehydrogenase mRNA level and in vitro chemosensitivity to 5-fluorouracil, in relation to differentiation in gastric cancer
}

Elham Fakhrejahani · Akiko Miyamoto •

Nobuhiko Tanigawa

Published online: 8 May 2007

(C) Springer-Verlag 2007

\section{Erratum to: Cancer Chemother Pharmacol}

\section{DOI 10.1007/s00280-007-0448-1}

Unfortunately, wrong version of Fig. 5 was printed. The corrected figure is given here.
The online version of the original article can be found at http://dx.doi.org/10.1007/s00280-007-0448-1.

E. Fakhrejahani - A. Miyamoto $\cdot$ N. Tanigawa $(\bowtie)$

Department of General and Gastroenterological Surgery,

Osaka Medical College, 2-7 Daigaku-cho,

Takatsuki-shi, Osaka 569-8686, Japan

e-mail: sur001@ poh.osaka-med.ac.jp
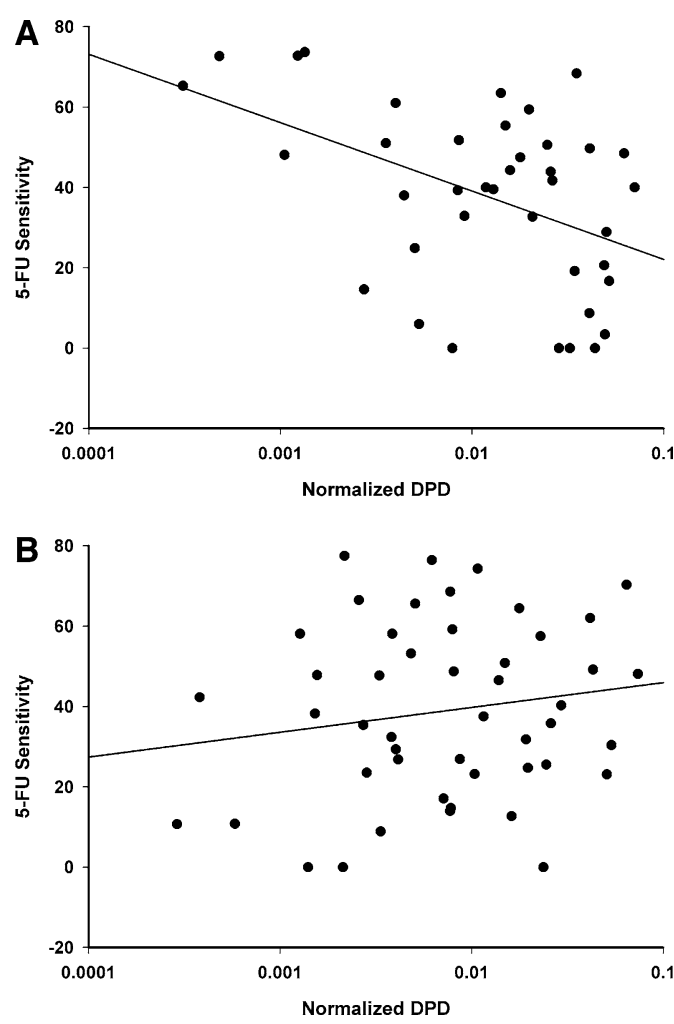

Fig. 5 Correlation between tumoral DPD levels and 5-FU sensitivity in undifferentiated (a) and differentiated group (b). Plots show the correlation between DPD: GAPDH RT-PCR product ratios of biopsied specimens and sensitivity to 5-FU measured by the MTT assay using formula: $\operatorname{IR}(\%)=(1-$ mean absorbance per gram of tumor specimen in the drug treated wells/mean absorbance per gram of tumor specimen in the non-drug treated control wells $) \times 100$. Spearman's rank correlation coeffcient was $r=-0.401$ in undifferentiated group and $r=0.126$ in differentiated group. DPD was a predictive factor for sensitivity only in undifferentiated group $(P=0.011)$. The correlation was not statistically significant in differentiated group 